\title{
Análise do Comportamento no Brasil ${ }^{1}$
}

\author{
João Claudio Todorov ${ }^{2}$ \\ Instituto de Educação Superior de Brasília (IESB) \\ Elenice S. Hanna \\ Universidade de Brasilia \\ Instituto Nacional de Ciência e Tecnologia sobre Comportamento, Cognição e Ensino
}

\begin{abstract}
RESUMO - O behaviorismo é uma filosofia da ciência preocupada com o tema e métodos da psicologia. Análise do comportamento não é uma área da psicologia, mas uma maneira de estudar o objeto da psicologia. No Brasil, a análise do comportamento começou com a vinda de Fred S. Keller para a Universidade de São Paulo e Universidade de Brasília nos anos 60. O Brasil é hoje o maior centro de análise do comportamento depois dos Estados Unidos e seus pesquisadores publicam nos melhores periódicos nacionais e internacionais. Os brasileiros pioneiros da análise do comportamento são responsáveis pelo reconhecimento da psicologia como profissão, pela fundação dos primeiros laboratórios no país e pela criação do Conselho de Psicologia e da Sociedade Brasileira de Psicologia.
\end{abstract}

Palavras-chave: análise do comportamento; behaviorismo; Fred S. Keller; Carolina M. Bori.

\section{Behavior Analysis in Brazil}

\begin{abstract}
Behaviorism is the philosophy of a science interested on the subject and method of psychology. Behavior analysis is not a field of psychology; it is a way to study the object of psychology. Behavior analysis in Brazil started when Fred S. Keller came to work at the University of São Paulo and the University of Brasília in the 60s. Brazil has the largest behavior analysis community outside the United States. Brazilian researchers publish in the best national and international journals. The pioneers in behavior analysis were also responsible for the recognition of psychology as a profession, the foundation of the first laboratories in the country, and the foundation of the Brazilian Council of Psychology and the Brazilian Psychological Society.
\end{abstract}

Keywords: behavior analysis; behaviorism; Fred S. Keller; Carolina M. Bori.

O presente artigo foi organizado em quatro partes. A primeira resume aspectos históricos, teóricos e metodológicos da análise do comportamento a partir de uma visão pessoal retratada em publicações anteriores do primeiro autor (Todorov, 1982, 1990, 1996, 2003, 2006). A segunda parte descreve a introdução da análise do comportamento no Brasil por Fred Keller e brasileiros pioneiros e relata como era a formação básica dos novos pesquisadores brasileiros em psicologia. A terceira parte apresenta o desenvolvimento do ensino de análise do comportamento no Brasil, especialmente na pós-graduação, e a quarta avalia parte da produção dos pesquisadores brasileiros relacionada à análise do comportamento, seu impacto e suas lacunas.

\section{Behaviorismo e Análise do Comportamento}

O comportamentalismo, com acentuação no 'ismo', não é o estudo cientifico do comportamento, mas uma filosofia da ciência preocupada com o tema e métodos da psicologia. (Skinner, 1969/1980, p. 339)

1 Agradecemos a Deisy das Graças de Souza e Marcelo L. Benvenuti por nos lembrarem de alguns nomes e acontecimentos importantes.

2 Endereço para correspondência: SHIN QI 01 Conjunto 09 Casa 11. Brasília, DF. CEP 71505-090.E-mail: joaoclaudio.todorov@gmail. com; elenicehanna@gmail.com.
A análise do comportamento não é uma área da psicologia, mas uma maneira de estudar o objeto da psicologia. Este trabalho tenta esclarecer os significados dos termos "behaviorismo", "análise do comportamento" e "psicologia". O termo "behaviorismo" tem sido utilizado de diversas maneiras e de tal modo que se pode afirmar que há muitas variedades de significado para ele. Desde o manifesto de Watson, muitas características foram atribuídas ao termo. Muitas delas perderamse no tempo ante críticas irrespondíveis, outras permanecem. Para Harzem e Miles (1978), a palavra behaviorismo tem uma "família de significados" e, por isso, além de desnecessário, é um equívoco esperar-se encontrar o seu "verdadeiro" significado. Portanto, a menos que se faça a distinção entre as diversas variedades de significados, não é útil proclamar-se "a favor" ou "contra" o behaviorismo.

Harzem e Miles (1978) utilizam uma classificação defendida por Mace (1948) para as variedades de behaviorismo: metafísico, metodológico e analítico. $\mathrm{O}$ behaviorismo metafísico afirma que mentes ou eventos mentais não existem; o behaviorismo metodológico afirma que se mente ou eventos mentais existem, não são objetos apropriados para o estudo científico; e o behaviorismo analítico afirma que os enunciados feitos com o propósito de se referir à mente ou eventos mentais tornam-se, quando analisados, enunciados acerca do comportamento. Harzem e Miles argumentam que as discussões sobre o behaviorismo metafísico e o behaviorismo metodológico são o resultado de erros conceituais, e 
que tanto a aceitação quanto a rejeição de um ou de outro são igualmente (e logicamente) injustificáveis. O behaviorismo analítico é diferente dos outros dois tipos porque suas proposições têm caráter claramente conceitual. A tese central afirma que sentenças a respeito de mentes e eventos mentais requerem uma tradução para sentenças sobre o comportamento. O behaviorismo analítico, nesse sentido, é uma proposta conceitual: não é uma teoria sobre o que deve ser estudado, nem é um conjunto de instruções sobre como se deve estudar, nem é um conjunto de instruções sobre como se deve fazer pesquisa (Harzem \& Miles, 1978).

A análise do comportamento origina-se de uma posição behaviorista assumida por Skinner (e.g., 1961, 1953/1967, 1974, 1957/1978, 1969/1980) por motivos mais históricos que puramente lógicos. Skinner parte da constatação de que há ordem e regularidade no comportamento. Um vago senso de ordem emerge da simples observação mais cuidadosa do comportamento humano. Estamos todos continuamente analisando circunstâncias e predizendo o que os outros farão nessas circunstâncias, e nos comportamos de acordo com nossas previsões. Se as interações entre os indivíduos fossem caóticas, simplesmente não estaríamos aqui. O estudo científico do comportamento aperfeiçoa e completa essa experiência comum quando demonstra mais e mais relações entre circunstâncias e comportamentos e quando demonstra as relações de forma mais precisa.

Para entender-se análise do comportamento, é necessário conhecer algumas das premissas sustentadas por Skinner e associados e aceitas por aqueles que se denominam analistas do comportamento. Vejamos algumas dessas premissas:

a) Os homens agem sobre o mundo, modificam-no e são modificados pelas conseqüências de suas ações. (Skinner, 1957/1978)

b) A psicologia é o estudo da interação entre organismo e ambiente. (Harzem \& Miles, 1978)

c) Através de análise, chega-se aos conceitos de estímulo e resposta. Um estímulo pode ser definido como 'uma parte, ou mudança em uma parte, do ambiente', já uma resposta pode ser definida como 'uma parte, ou mudança em uma parte, do comportamento. No entanto, um estímulo não pode ser definido independentemente de uma resposta. (Keller \& Schoenfeld, 1950/1968)

Com esses pressupostos, e sem descartar a priori quaisquer fontes de informação, a análise do comportamento desenvolveu-se como uma linguagem da psicologia, aperfeiçoou métodos de estudo para questões tradicionais da psicologia, abriu novos campos de pesquisa e gerou tecnologias em uso por toda parte. Já se escreveu muito sobre os métodos da análise do comportamento e as descrições são aproximadamente as mesmas, variando apenas na ênfase dada a estes ou aqueles aspectos (e.g., Honig, 1966; Honig \& Staddon, 1977; Lattal \& Chase, 2003; Skinner, 1961, 1969/1980). Tais métodos são utilizados por um grupo de pesquisadores, um grupo de dimensões razoáveis, que vem crescendo desde os anos 50 do século passado.

Como resumido por Honig (1966), os métodos de trabalho na pesquisa caracterizam-se pela utilização conjunta dos seguintes aspectos quando o trabalho é de análise experimental:
(1) estudo intensivo do comportamento do indivíduo; (2) controle estrito do ambiente experimental; (3) uso de uma resposta repetitiva que produz pouco efeito imediato no ambiente; (4) meios eficazes de controle do comportamento do sujeito; (5) observação e registro contínuo do comportamento; e (6) programação de estímulos e registro de eventos automáticos.

É interessante notar que as características dos métodos utilizados geralmente referem-se apenas à análise experimental do comportamento de animais não humanos. Essa caracterização é falha, e por vários motivos. Primeiro, não há sentido em descrições que confundam análise do comportamento com análise experimental do comportamento de animais não humanos. Ao apontar as virtudes dessas descrições, Skinner (1953/1967) foi claro:

O comportamento humano se caracteriza por sua complexidade, sua variedade, e pelas suas maiores realizações, mas os princípios básicos não são por isso necessariamente diferentes. A ciência avança do simples para o complexo: constantemente tem que decidir se os processos e leis descobertos para um estágio são adequados para o seguinte. Seria precipitado afirmar neste momento que não há diferença essencial entre o comportamento humano e o comportamento de espécies inferiores; mas até que se empreenda a tentativa de tratar com ambos nos mesmos termos, seria igualmente precipitado afirmar que há. (p. 47)

Uma análise experimental do comportamento de animais não humanos é, então, uma parte, e não necessariamente a inicial, do trabalho. Ela também não é um fim em si mesma.

Segundo, as caracterizações normalmente ignoram análise conceitual como parte de uma análise do comportamento. Entretanto, é fácil constatar o quanto da contribuição de Skinner à psicologia tem a ver com o que Harzem e Miles (1978) denominam "o comportamento lógico dos conceitos". Veja-se, por exemplo, além de Ciência e Comportamento Humano (Skinner, 1953/1967), About Behaviorism (Skinner, 1974) e os trabalhos de Evalyn Segal (1977), George Robinson (1977), Emílio Ribes-Iñesta (2003), Jack Michael (2004) e Sigrid Glenn (2003).

Terceiro, muito do progresso obtido pela análise do comportamento deve-se a análises funcionais não experimentais. Seguramente, mais da metade dos escritos de Skinner referese a análises funcionais não-experimentais, isto é, à identificação (ou tentativa de identificação) de variáveis dependentes e independentes e de processos de interação em exemplos de comportamento humano. Veja-se, como ilustração, as seções "O indivíduo como um todo", "O comportamento de pessoas em grupo" e "Agências controladoras", em Ciência e Comportamento Humano (Skinner, 1953/1967) e o livro Contingências de Reforço (Skinner, 1969/1980).

Quarto, intentos de caracterização da análise do comportamento muitas vezes confundem aspectos da análise com idiossincrasias do analista. Os trabalhos de Skinner, por exemplo, podem ser vistos sob diferentes prismas: há trabalhos de análise experimental, de análise conceitual, de análise funcional não experimental e há trabalhos de prescrição moral. Poucos analistas do comportamento admitiriam, entretanto, que prescrições morais caracterizam a análise do comportamento. 
Quinto, questões ideológicas muitas vezes são confundidas com caracterizações da análise do comportamento, especialmente quando o aspecto ideológico não é explicitado. Vale ressaltar que isso quase sempre acontece quando se discute a resolução de problemas práticos por psicólogos que se utilizam de uma análise do comportamento. Neste ponto, devemos admitir que a ideologia dominante em uma sociedade dirige tanto os esforços de pesquisa quanto os de aplicação. Quando questões ideológicas não são explicitadas e analisadas, corremos o risco de confundir pressupostos básicos da análise do comportamento com características ideológicas de uma determinada sociedade.

Quando Skinner (e.g., 1953/1967) explicitou um programa de trabalho para o desenvolvimento de uma ciência do comportamento, previu uma análise experimental do comportamento como um dos aspectos de um empreendimento maior. Para Skinner, o material a ser analisado provém de muitas fontes, das quais a análise experimental do comportamento é apenas uma delas. Skinner aponta a utilidade de observações casuais, observações de campo controlada, observações clínicas, observações controladas do comportamento em instituições, estudos em laboratório do comportamento humano e, por fim, estudos de laboratório do comportamento de animais não humanos. Não há sentido, pois, em discutir análise experimental do comportamento sem primeiro discutir análise do comportamento.

Vejamos, então, um resumo do exposto. Behaviorismo analítico (ou linguístico, como prefeririam os filósofos de hoje) é uma reflexão a respeito dos enunciados da psicologia: não é uma teoria sobre o que deve ser estudado, nem é um conjunto de instruções sobre como se deve fazer pesquisa. A análise do comportamento é uma linguagem da psicologia que tem como seu objeto o estudo de interações comportamento-ambiente. Interessa-se, especialmente, pelo homem, mas estuda também interações envolvendo outros animais sempre que houver algum motivo para supor que tais estudos possam ajudar no esclarecimento de interações homem-ambiente. A análise experimental do comportamento busca relações funcionais entre variáveis, controlando condições experimentais (variáveis de contexto, segundo Staddon, 1973), manipulando variáveis independentes (mudanças no ambiente) e observando os efeitos em variáveis dependentes (mudanças no comportamento).

O conceito de ambiente é decomposto em histórico, biológico, físico e social apenas como um recurso de análise útil para apontar os diversos fatores que, indissociáveis, participam das interações estudadas pelo psicólogo. Sem a decomposição necessária para a análise, o todo é ininteligível; por outro lado, a ênfase exclusiva nas partes pode levar a um conhecimento não relacionado ao todo. O jogo constante de ir e vir, de atentar para a intercalação das partes na composição do todo, é essencial para o entendimento das interações organismo-ambiente.

Assim como o ambiente pode ser analisado em diferentes níveis, comportamento pode ser entendido em diferentes graus de complexidade. Não é a quantidade ou a qualidade de músculos ou glândulas envolvidas, ou os movimentos executados, o que importa. O comportamento não pode ser entendido isolado do contexto em que ocorre. Não há sentido em uma descrição de comportamento sem referência ao ambiente, como não há sentido, para a psicologia, em uma descrição do ambiente apenas. Os conceitos de comportamento e ambiente, e de resposta e estímulo, são interdependentes. Um não pode ser definido sem referência ao outro (Todorov, 1981). Sobre a questão da definição de comportamento, Schick (1971) afirma:

Quando nos lançamos a construir uma ciência do comportamento, somos imediatamente confrontados por dois problemas. O primeiro problema é o de dizer quanto do que ocorre no mundo é considerado comportamento. Todas as mudanças em estados dos organismos são comportamentais, ou apenas parte delas? Se apenas parte delas, então quais?... O segundo problema é o de selecionar unidades de comportamento. Como deve o comportamento ser dividido em unidades, de maneira a tornar possível uma explicação?... Felizmente, não é necessário afirmar exatamente o que é comportamento antes de iniciarmos a construção de uma ciência do comportamento. Podemos isolar algumas instâncias do comportamento e começar a estudá-las, mesmo que não seja possivel definir exaustivamente o que é eo que não é comportamento. (p. 413, tradução livre dos autores)

Ao isolar alguma instância do comportamento, estamos detectando algum tipo de interação organismo-ambiente. Vejamos um exemplo. A perda de um parente próximo será seguida ou não de depressão dependendo de fatores como a idade de quem morreu, a idade do parente que sobrevive, o grau de parentesco, o grau de afetividade do relacionamento, a duração da enfermidade, a magnitude da herança etc. Ou seja, a relação funcional "perda de um parente-depressão exógena" depende de variáveis de contexto, que são os fatores acima apontados (Staddon, 1973). Staddon, em um artigo sobre a noção de causa em psicologia, mostra como a noção de contexto não é limitada temporalmente. Contexto não se refere apenas às características atuais do ambiente externo. No nosso exemplo, o grau de afetividade pode ter se estabelecido há anos por meio de interações envolvendo os dois parentes e exerce sua influência mesmo que a morte ocorra num período em que os dois não se comunicam há muito tempo.

O exemplo serve para ilustrar a arbitrariedade na escolha do que é causa e do que é contexto. Poderíamos falar da relação funcional "grau de parentesco-depressão", dado o contexto da morte de um parente próximo. A seleção de uma variável como causa e a designação de outras como contexto vai depender de quais são os interesses envolvidos no estudo, pois quando variáveis de contexto são consideradas, uma relação de causa e efeito é apenas um instrumento para a descoberta de princípios de maior generalidade. Princípios são a descrição mais econômica do conjunto de relações causais e variáveis de contexto que dão origem a eles. Um sistema de relações funcionais bem definidas resultará em uma teoria útil se também vier acompanhada de especificações de onde, no ambiente externo, as variáveis independentes e as variáveis de contexto devem ser encontradas, além de instruções sobre como detectá-las e/ou medi-las. Causas, pois, são os ingredientes primários e empíricos com os quais se constroem explicações (teorias) mais abrangentes. Portanto, o termo "causa" tem sentido apenas dentro de uma teoria ou modelo. Não há uma causa real de um dado evento. Há apenas mode- 
los do mundo mais ou menos adequados, e sempre passíveis de modificação, de acordo com critérios como predição, simplicidade e generalidade, entre outros (Staddon, 1973).

Para a identificação de relações funcionais, o analista do comportamento se utiliza do conceito de contingência como instrumento. $O$ termo contingência é empregado para se referir a regras que especificam relações entre eventos ambientais ou entre comportamento e eventos ambientais (Skinner, 1953/1967). O enunciado de uma contingência é expresso em forma de afirmações do tipo "Se, então". A cláusula "se" pode especificar algum aspecto do comportamento (Weingarten \& Mechner, 1966) ou do ambiente (Schwartz \& Gamzu, 1977), e a cláusula "então" especifica o evento ambiental consequente. Assim como relações funcionais são instrumentos na busca de princípios mais gerais, contingências são utilizadas pelo psicólogo experimental na procura de relações funcionais. As contingências são as definições de variáveis independentes na análise experimental de comportamento.

A análise do comportamento tem algumas características que a distinguem de outras linguagens que prosperam na psicologia. Dentre essas características, pode-se citar a análise experimental do comportamento de organismos individuais $(\mathrm{n}=1)$. Entretanto, essa análise, quer seja realizada no laboratório, quer no consultório clínico, não seria sua marca mais distinta; sua linguagem teórica o seria. De fato, a linguagem teórica da análise do comportamento é o cimento que une todos os tipos de atividades compreendidas sob essa rubrica.

Experimentação com $n=1$ é a grande contribuição de Skinner para a psicologia experimental dos anos 30 do século passado. Skinner a trouxe de seus estágios nos principais laboratórios de biologia da Universidade de Harvard. Junto com a taxa de respostas (número de respostas por unidade de tempo) e os esquemas de reforço intermitente, forma o trio de ouro de Skinner. No entanto, nem Skinner ficou só na análise experimental do comportamento de organismos individuais ( $\mathrm{n}=1)$. Já em Ciência e Comportamento Humano (1953/1967), ele mostrou como se pode avançar analisando exemplos da vida diária à luz da teoria. E é essa teoria, que começou a ser desenvolvida em O Comportamento dos Organismos (1938), continua sendo desenvolvida até hoje e continuará a ser desenvolvida pela futuras gerações, que faz a conexão entre os diferentes campos de atuação da análise do comportamento: pesquisa básica, pesquisa aplicada, atuação profissional, análise conceitual etc.

O triângulo com teoria em uma ponta, pesquisa básica na outra e pesquisa aplicada na terceira, com comunicação de mão dupla entre as três pontas, é uma representação frequentemente utilizada, mas que é útil talvez apenas para justificar decisões tomadas no passado longínquo e que nos atrapalham até hoje. Em vez do triângulo, um $\mathbf{V}$ invertido, com a teoria acima, no vértice, que se comunica em mão dupla com os dois lados, o da pesquisa e o da atuação profissional, seria a representação mais adequada na maioria dos casos. Não há comunicação entre pesquisa e atuação profissional a não ser via vértice, a teoria. Da mesma forma, a atuação profissional alimenta a pesquisa via teoria.

Resumindo, a análise experimental do comportamento utiliza-se de contingências e de relações funcionais como instrumentos para o estudo de interações comportamentoambiente (Todorov, 1989/2007). O experimentador mani- pula contingências em busca de relações funcionais e das condições (variáveis de contexto) nas quais essas relações podem ser observadas (Todorov, 1991). Um sistema de relações funcionais constituirá uma teoria útil se vier acompanhado de especificações de onde, no ambiente externo, as variáveis independentes e as variáveis de contexto devem ser encontradas.

Interações comportamento-ambiente ocorrem sempre no tempo. Nosso objeto de estudo não é uma coisa, mas um processo. A maior importância dada aos esforços de quantificação, nos últimos tempos, tem levado os estudos de análise experimental do comportamento a uma preocupação com processos estáveis. Para a análise do comportamento e, em especial, para a resolução de problemas práticos pelo psicólogo, interessam também, e muito, as informações sobre processos em estágio de transição (Todorov, 1983). Quando uma nova contingência entra em vigor, seus efeitos dependerão dos processos de interação que estão ocorrendo. O psicólogo experimental pode dispor as condições mais adequadas (ou menos inadequadas) para estudar essas transições e também para estudar interações dos efeitos de diversas variáveis. $\mathrm{O}$ psicólogo no exercício profissional, entretanto, defronta-se com um problema prático. A identificação dos processos de interação quase nunca pode ser feita após minucioso estudo experimental acerca de quais variáveis, dentre as diversas possíveis, estão presentes no caso que tem em mãos. O psicólogo depende, nessas circunstâncias, da linguagem teórica da análise do comportamento para orientá-lo na identificação dos processos e nas possíveis intervenções. Ao refletir sobre essa linguagem e sobre sua aplicabilidade à realidade em que se vive, o psicólogo contribui para os trabalhos de análise conceitual e de revisão conceitual (Todorov, 1987; Todorov \& Moreira, 2004).

\section{Fred Keller e a Análise do Comportamento no Brasil}

A amizade de B. F. Skinner e Fred Keller foi crucial para o desenvolvimento intelectual e científico de ambos. O livro Princípios de Psicologia (Keller \& Schoenfeld, 1950/1968) foi um marco na história da análise do comportamento, tendo preparado o terreno para Ciência e Comportamento Humano (Skinner, 1953/1967). Naturalmente, esses livros foram os primeiros a serem traduzidos para o português. A importância desses livros para a introdução do behaviorismo no Brasil foi diversas vezes reconhecida (e.g., Todorov, 1990) por ocasião do $40^{\circ}$ aniversário da publicação de Princípios de Psicologia, do centenário do nascimento de B. F. Skinner e do $70^{\circ}$ aniversário da publicação de $O$ Comportamento dos Organismos.

Fred S. KeIIer veio ao Brasil em 1961, como um Fulbright Scholar, para ensinar durante um ano na Universidade de São Paulo (USP). Naquele ano, só a Pontifícia Universidade Católica do Rio de Janeiro (PUC-RJ) e a USP ofereciam o curso de graduação em psicologia, iniciados os dois em 1958 (o primeiro da América Latina começou em 1947, na Universidad Nacional de Colombia, em Bogotá). A Faculdade de Filosofia, Ciências e Letras da USP tinha menos que 30 anos e ainda respirava a marcada influência dos professores 
europeus que vieram nos anos 30. A influência americana começou durante a II Guerra Mundial, e a Comissão Fulbright no MEC era parte de um acordo entre os governos do Brasil e dos EEUU. Entre todos os professores de psicologia que Keller conheceu na USP, só Carolina Martuscelli Bori tinha estudado nos Estados Unidos, onde concluiu o mestrado em psicologia social na New School for Social Research of New York. Nessa época, Skinner era um desconhecido no Brasil. Os autores estudados eram todos europeus: Freud, Piaget, Kurt Lewin, Anastasi, Moreno, Klinenberg, entre muitos outros.

$\mathrm{Na}$ USP, Keller ensinou o primeiro curso de análise do comportamento da América Latina, na disciplina Psicologia Experimental, oferecida no terceiro ano da graduação. Esse curso tinha o objetivo de dar aos problemas da psicologia moderna um tratamento experimental do ponto de vista da teoria do reforçamento (Keller, 1987). Com Carolina Bori and Rodolpho Azzi como professores assistentes de Fred Keller, os primeiros alunos foram Maria Amélia Matos, Dora Fix Ventura, Mário Guidi, Margarida Windholz, Vera Konigsberger, Maria Inês Rocha e Silva, entre outros (Bori, 1996; Keller, 1981; Matos, 1996, 1998). Trabalhando no Departamento de Fisiologia da faculdade por falta de laboratório no Departamento de Psicologia, sem equipamentos adequados e em precárias condições, Keller conseguiu ensinar com exercícios práticos, concluindo um experimento sobre o efeito do atraso de reforço na aprendizagem, posteriormente publicado no Journal of the Experimental Analysis of Behavior (JEAB) (Azzi, Fix, Keller \& Rocha e Silva, 1964). O experimento foi realizado com uma gaiola de pássaros como câmara experimental, o rato ficava abrigado em outra gaiola, a tradicional barra de metal foi substituída por um arame, nada era elétrico nem automático, mas foi possível usar água como reforço (Kerbauy, 1983). Nesse primeiro ano, Keller ainda trabalhou no primeiro dicionário inglês-português da terminologia operante, primeiro passo em uma longa sequência de traduções de artigos e livros (Azzi, Rocha e Silva, Bori, Fix, \& Keller, 1963).

$\mathrm{O}$ que aconteceu antes e depois dessa viagem está fartamente documentado (e.g., Bori, 1996; Gorayeb, 1996; Guilhardi \& Madi, 1996; Keller, 1968, 1971, 1982, 1987; Kerbauy, 1983; Matos, 1996; Pessotti, 1996; Stamirowski, 1998; J. C. Todorov, 1990, 1996, 2003; M. S. R. Todorov, 1995, 1997; Zannon, 1996; Zannon \& Bori, 1996). O sucesso de seu trabalho levou ao convite de um segundo Fulbright Scholar, John Gilmour (Gil) Sherman, na época um jovem PhD da Universidade de Columbia. Gil Sherman, Keller, Carolina Bori e Rodolpho Azzi, convidados pela Universidade de Brasília (UnB), planejaram e começaram a implantar o curso de psicologia nessa universidade, incluindo o desenvolvimento $^{3}$ e a aplicação do Sistema Personalizado de Ensino - PSI (Keller, 1968, 1982, 1987). Em março de 1963, os planos para um departamento de psicologia em Brasília foram discutidos com Keller em Nova Iorque.

3 Um protótipo do PSI foi desenvolvido por Isaias Pessotti e Herma Drachenberg na Faculdade de Filosofia Ciências e Letras de Rio Claro, em 1962, sob a supervisão de Carolina Bori, a partir de suas discussões com Fred Keller sobre a nova modalidade de ensino.
A introdução dos alunos à análise experimental do comportamento foi planejada como um curso de dois semestres. O primeiro semestre (IAEC 1) era basicamente o curso como originalmente planejado por Keller e Schoenfeld na Universidade de Columbia, usando o livro Princípios de Psicologia como texto. O curso foi adaptado para um sistema personalizado de ensino por Keller e Gil Sherman e testado naquela universidade em 1963. O PSI foi, a seguir, completamente desenvolvido pelos quatro autores que discutiram em Nova Iorque a ideia original (Gil Sherman, Keller, Carolina Martuscelli Bori e Rodolpho Azzi) e começou a ser implantado na UnB em agosto de 1964. O segundo semestre do curso introdutório, o IAEC 2, incluía apenas leituras, demonstrações e experimentos relacionados ao comportamento humano, com traduções para o português de alguns dos primeiros (e agora clássicos) trabalhos de análise experimental do comportamento humano. Entre esses, estavam os estudos de Greenspoon sobre comportamento verbal (Greenspoon, 1955), o condicionamento de respostas textuais (Staats, Staats, Schultz \& Wolf, 1962), o uso de fichas como reforçadores (Ayllon \& Michael, 1959) e trechos de Experimental Foundations of Clinical Psychology (Bachrach, 1962).

Em janeiro de 1963, Todorov junta-se ao grupo como instrutor (auxiliar de ensino). Enquanto ainda estava em São Paulo, seus deveres incluíam a tradução de material a ser usado no curso em Brasília. Science and Human Behavior (Skinner, 1953) foi parte desse material. Todorov traduzia os textos e os passava para Rodolpho Azzi, tradutor experimentado, que fazia a revisão. Ciência e Comportamento Humano estava pronto no final de 1964, usando os termos técnicos anteriormente traduzidos e publicados no $J E A B$ (Azzi \& cols., 1963), uma das muitas iniciativas de Keller em seu primeiro ano no Brasil. A tradução foi um trabalho fascinante para Todorov, recém saído da graduação, uma vez que o livro cobria a maior parte do campo da psicologia com o qual o aluno tinha contato, por meio de diferentes linguagens teóricas, durante o curso na USP. Ciência e Comportamento Humano foi a última contribuição do primeiro autor para o curso de introdução à análise experimental do comportamento na UnB, porque de janeiro a julho de 1965 ele se dedicou a coletar e analisar dados, e escrever a sua tese de mestrado, antes de viajar para a Arizona State University, para o doutorado que começaria em setembro.

Em outubro de 1965, a UnB passou por uma das maiores crises que a afetaram durante a ditadura militar. Nove professores de diversos departamentos, incluindo Rodolpho Azzi, foram demitidos por motivos políticos. Em protesto, mais de 200 professores, representando mais de $90 \%$ do corpo docente da UnB, pediram demissão. Dos professores de psicologia então em exercício, só Robert Berryman permaneceu. Fred Keller e Gil Sherman estavam em Tempe, na Arizona State University, desde agosto de 1964. James e Jean Nazzaro voltaram aos Estados Unidos em julho de 1965. Alunos do curso de mestrado foram contratados para substituir os demissionários (M. S. R. Todorov, 1997, Tabela 3). O grupo original liderado por Fred Keller e Carolina Bori se dissolveu, mas o IAEC 1, o semestre introdutório para o qual o PSI foi desenvolvido, sobreviveu. Hoje em quase todos os cursos de psicologia no Brasil há alguma versão dele. 


\section{Formação em Análise do Comportamento no Brasil}

O primeiro centro de formação de analistas do comportamento foi a Universidade de Brasília (Todorov, 2006) (e continua muito produtivo até hoje), sob o comando de Carolina Bori. A partir de $1970^{4}$, de volta a São Paulo, Carolina Bori comanda, na Universidade de São Paulo, o maior centro de formação em análise do comportamento, na Universidade de São Paulo (incluindo o campus de Ribeirão Preto), até os anos 90, quando se consolidam os cursos da Pontifícia Universidade Católica de São Paulo (PUC-SP), da Universidade Federal de São Carlos (UFSCar), da Universidade Federal do Pará (UFPA), da Universidade Federal de Santa Catarina (UFSC) e da Universidade Estadual de Londrina (UEL), todas influenciadas direta ou indiretamente por São Paulo e/ou Brasília. A formação básica do psicólogo brasileiro sofreu grande influência da atuação de Carolina Bori. Ela foi a principal responsável pela criação e disseminação de laboratórios de ensino em psicologia experimental no país (Matos, 1998). Graças aos esforços de Carolina Bori, todos os cursos de graduação de grandes centros de formação passaram a incluir em seus currículos, e mantêm até hoje, o conteúdo de psicologia como ciência natural, a preocupação com a observação sistemática e os métodos de investigação científica, bem como demonstrações, em atividades de laboratório, de manipulação e controle de variáveis ambientais e de seus efeitos sobre o comportamento.

A análise do comportamento permanece na formação do aluno de graduação nas disciplinas de Psicologia da Aprendizagem e Psicologia Geral Experimental, que faziam parte do currículo mínimo. A análise do comportamento é também ensinada em contextos aplicados, como nas disciplinas de Psicologia do Excepcional e Psicologia Clínica, mas isso ocorre em um número menor de instituições de ensino superior. Os progressos obtidos nas últimas décadas pelos analistas do comportamento em temas como ensino-aprendizagem, autismo, psicologia organizacional, psicologia dos esportes, psicologia da saúde e psicologia social, não produziram uma ampliação do contato do aluno com a análise do comportamento, que continua sendo ensinada em apenas uma ou duas disciplinas nos primeiros semestres do curso. Em muitas instituições, as informações sobre análise do comportamento, durante os cinco anos do curso de psicologia, ainda hoje se restringem aos conceitos básicos de reforçamento, punição e esquemas. Utiliza-se com frequência apenas os primeiros capítulos de Ciência e Comportamento Humano, juntamente com alguns capítulos de Princípios de Psicologia ou de algum de seus sucedâneos (Baum, 1994/1999; Catania, 1998/1999; Millenson, 1967), em um curso de um semestre apenas. Mais recentemente, tem crescido o uso de um livro

4 Com a diáspora de Brasília, professores e instrutores se espalharam pelo país dando origem a novos centros de difusão e ensino de análise do comportamento. Nessa, época Luiz de Oliveira já estava trabalhando na FFCLRP e Luiz Otavio Queiroz na PUCCAMP. O primeiro começou uma tradição em pesquisa básica aproximando análise do comportamento e biologia, especialmente nutrição e farmacologia. O segundo começou em Campinas o primeiro centro voltado para a formação de profissionais, sendo Helio Guilhardi e Maria Helena Leite Hunziker uns dos primeiros alunos. novo de autores brasileiros, Princípios Básicos de Análise do Comportamento (Moreira \& Medeiros, 2007).

Outras partes de Ciência e Comportamento Humano são usadas, contudo, em diversas outras disciplinas, da Psicologia do Desenvolvimento à Psicologia Organizacional. À medida que a análise de práticas culturais torna-se matéria de interesse dos analistas do comportamento (Biglan, 1995; Guerin, 1994; Lamal, 1997; Sidman, 1989/1998), Ciência e Comportamento Humano continuará a ser uma inspiração para os interessados em todos os aspectos do comportamento social humano. $\mathrm{Na}$ verdade, as seções sobre as agências controladoras são mais relevantes agora do que no século passado. Skinner estava escrevendo sobre o governo durante os primeiros anos da Guerra Fria. É interessante notar que, ao contrário de obras de ficção da época sobre governos totalitários (Orwell, 1949, por exemplo), Skinner analisa controles e contra-controles durante o funcionamento imperfeito de sistemas democráticos - imperfeitos na medida em que um sistema democrático envolve um balanceamento contínuo de controles e contra-controles. As ditaduras, por outro lado, desenvolvem todos os esforços para tornar difícil o contra-controle.

Mais do que a quantidade de disciplinas onde se ensina exclusivamente análise do comportamento, nosso desafio é integrar a análise do comportamento aos diferentes temas da psicologia. Um segundo desafio do grupo é evitar que as informações sejam ensinadas, como ocorre em vários cursos de psicologia e áreas afins, por profissionais que desconhecem inclusive os seus pressupostos, gerando nos alunos preconceito e posições equivocadas sobre a proposta da análise do comportamento.

A formação de qualidade proporcionada pelos centros influenciados por Fred Keller, Carolina Bori e seus brilhantes assistentes, embora não esteja adequadamente configurada nos cursos de graduação, foi crucial para a criação dos cursos de mestrado e doutorado que formam pesquisadores de alto nível em diversas universidades brasileiras.

$\mathrm{Na}$ região sul, a UEL oferece mestrado em análise do comportamento, com linhas de pesquisa sobre análise teórico-conceitual, modelos experimentais e metodologia e tecnologia de intervenção em diferentes contextos. A UFSC possui cursos de mestrado e doutorado com possibilidade de orientação em análise do comportamento em organizações, trabalho e aprendizagem e em cognição. Existe também a possibilidade de orientação de mestrado em "Fundamentos conceituais do behaviorismo radical: epistemologia, ética e cultura" na Universidade Federal do Paraná (UFPR) e em estudos sobre uso e abuso de drogas e em epistemologia e história da psicologia na Universidade Federal do Rio Grande do Sul (UFRGS).

No sudeste, existem quatro IES e seis cursos de pósgraduação com pelo menos uma linha de pesquisa em análise do comportamento. A USP, campus de São Paulo, forma mestres e doutores em psicologia experimental, tendo como uma das linhas de pesquisa "Análise do comportamento". No campus de Ribeirão Preto, os cursos de mestrado e doutorado em psicologia possuem orientadores com formação em análise do comportamento na linha de pesquisa "Saúdedoença: prevenção, promoção e avaliação ". A PUC-SP forma mestres e doutores em "Psicologia Experimental - Análise 
do Comportamento", oferecendo linhas de pesquisa sobre história e fundamentos epistemológicos, metodológicos e conceituais, processos básicos, desenvolvimento de metodologia e tecnologias de intervenção. Também forma mestres a Universidade Estadual Paulista Júlio de Mesquita (UNESP), com duas linhas de pesquisa: "Aprendizagem e ensino" e "Desenvolvimento, comportamento e saúde". Os cursos de mestrado e doutorado em ensino especial da UFSCar possuem grande tradição na formação com base na análise do comportamento e oferecem quatro linhas de pesquisa relacionadas: "Aprendizagem e cognição de indivíduos com necessidades especiais de ensino"; "Currículo funcional: implementação e avaliação de programas alternativos de ensino especial"; "Práticas educativas: processos e problemas"; e "Atenção primária e secundária em Educação Especial: prevenção de deficiências". A UFSCar criou recentemente os cursos de mestrado e doutorado em Psicologia com uma linha de pesquisa "Análise comportamental da cognição" com três eixos: função simbólica, aplicações ao ensino e desenvolvimento de instrumentos de avaliação, recursos tecnológicos e material instrucional.

No centro-oeste, a UnB oferece cursos de mestrado e doutorado em ciências do comportamento, com duas áreas de concentração, sendo uma delas "Análise do comportamento", e com linhas de pesquisa sobre processos comportamentais básicos e análise comportamental aplicada. Na PUC-Goiás, há também a possibilidade de formação de mestrado e doutorado em psicologia com base comportamental, nas linhas de pesquisa "Análise e evolução do comportamento" e "Psicopatologia clínica e "Psicologia da saúde".

Na região nordeste, desconhecemos cursos de pós-graduação com possibilidade de orientação em análise do comportamento. Na região norte, a UFPA é o centro de referência, com cursos de mestrado e doutorado em "Teoria e pesquisa do comportamento", que oferecem linhas de pesquisa sobre processos psicológicos básicos, questões históricas e conceituais e desenvolvimento de tecnologia comportamental.

\section{Produção dos Analistas do Comportamento Brasileiros}

O grupo de analistas do comportamento cresceu muito no Brasil desde a vinda de Fred Keller. Os encontros da Associação Brasileira de Psicoterapia e Medicina Comportamental (ABPMC), que congrega pesquisadores, profissionais e alunos, recebiam em torno de 140 inscrições no início dos anos 90 do século passado e agora recebem cerca de 1500 inscrições. A ABPMC, atualmente sob a presidência de Maria Martha Hübner, possui uma divisão regional criada recentemente em Brasília (DBAC). Atualmente, 13 estados brasileiros de todas as regiões do país promovem encontros ou jornadas de análise do comportamento, oferecendo cursos, palestras e divulgando a pesquisa científica realizada no país.

Os analistas do comportamento foram também essenciais para a criação do conselho de psicologia e de sociedades de psicologia no Brasil. A atual Sociedade Brasileira de Psicologia (SBP), criada em 1970 como Sociedade de Psicologia de Ribeirão Preto (SPRP), foi criada e teve suas primeiras reuniões organizadas por presidentes científicos que eram analistas do comportamento (entre eles Todorov e Ricardo Gorayeb). Dos 18 presidentes que a SBP teve até hoje, $50 \%$ são analistas do comportamento. Essas reuniões foram os principais fóruns de discussão e divulgação científica da análise do comportamento no Brasil e, apesar da criação da ABPMC, a SBP continua até hoje sendo um espaço importante de intercâmbio científico do grupo.

Com relação à produção intelectual, o primeiro trabalho de análise experimental do comportamento feito no Brasil, conforme dito anteriormente, foi publicado no JEAB (Azzi $\&$ cols., 1964), enquanto o primeiro desenvolvido em Brasília foi publicado no Journal of Experimental Psychology (Nazzaro \& Todorov, 1966). A USP-RP foi, no final da década de 60 e início da década de 70 do século passado, um centro de pesquisa em controle aversivo liderado por J. C. Todorov. Nessa época, Deisy das Graças de Souza era bolsista de iniciação científica e Antonio Bento Alves de Moraes, Elenice Ferrari e Ricardo Gorayeb realizavam suas teses de mestrado. Os trabalhos, reconhecidos pela qualidade e originalidade, foram publicados em revistas internacionais (de Souza, Moraes \& Todorov, 1984; Ferrari, Todorov \& de Souza, 1974; Ferrari, Todorov \& Graeff, 1973; Gorayeb \& Todorov, 1977; Moraes \& Todorov, 1977). Esses trabalhos foram inicialmente motivados por questionamentos sobre a generalidade dos resultados obtidos com animais, especialmente pombos, baseados na dificuldade de se estabelecer a resposta de bicar como esquiva. Os resultados dos estudos comprovaram a sensibilidade do bicar às contingências aversivas.

A pesquisa, a partir de meados dos anos 70 e nos anos 80 do século passado, foi dominada por investigações sobre esquemas de reforçamento, comportamento de escolha e quantificação da lei do efeito (e.g., Todorov, 1971, 1973), com o grupo da UnB liderado por J.C. Todorov que interagia com M.L.D Ferrara e seus alunos da USP. Parte da pesquisa foi produzda ou publicada no México como resultado do ano em que Todorov foi Professor Visitante da Universidad Nacional Autonoma do Mexico (e.g., Todorov, 1977, 1979). A motivação para estudar escolha foi gerada pelo artigo seminal de Herrnstein (1970), cuja tradução será brevemente publicada pela Revista Brasileira de Análise do Comportamento.

As visitas de Murray Sidman nos anos 80 e 90 do século passado às universidades brasileiras e a divulgação da relação entre comportamento simbólico e seu modelo sobre equivalência de estímulos (Sidman \& Tailby, 1982) modificaram o perfil da pesquisa no Brasil. Deisy das Graças de Souza e Julio C. Coelho de Rose, da UFSCar, iniciaram interações com pesquisadores de diferentes universidades brasileiras (USP-SP, UFPA, UnB, UNESP) e americana (University of Massachussets Medical School). Essas interações científicas culminaram na aprovação do primeiro Núcleo de Excelência em Pesquisa da psicologia, coordenado por Júlio de Rose no período 1998-2003 e do primeiro Instituto Nacional de Ciência e Tecnologia (INCT) da psicologia, coordenado por Deisy no período 2008-2011. O INCT constitui-se em uma rede multi-institucional e multirregional, com foco na ciência e tecnologia comportamental, que trata do funcionamento simbólico e de seus déficits funcionais, particularmente em crianças. $\mathrm{O}$ programa de pesquisa, construído de forma inovadora, possui um componente de ciência básica (voltado para a compreen- 
são de aspectos elementares do funcionamento simbólico e desenvolvimento de procedimentos potencialmente úteis na prevenção ou redução de déficits de função simbólica); um componente de ciência translacional (voltado para a validação efetiva de novos princípios e/ou novos procedimentos derivados de estudos clínico/educacionais iniciais, em condições quase-controladas); e um componente de ciência aplicada (voltado para o desenvolvimento de soluções para os desafios educacionais e terapêuticos em escolas, hospitais, clínicas etc). Compõem o INCT sobre Comportamento, Cognição e Ensino, pesquisadores da Escola Experimental de Primatas da UFPA, coordenada por Olavo F. Galvão e Romariz da Silva Barros. Esse laboratório desenvolve estudos com o objetivo de identificar a rota de ensino e os procedimentos necessários para a obtenção de classes de estímulos equivalentes em animais não-humanos, principalmente primatas. As diversas contribuições científicas desenvolvidas nesse laboratório têm sido divulgadas amplamente em revistas nacionais e internacionais (e.g., Barros \& Galvão, 2002; Souza, Borges, Goulart, Barros \& Galvão, 2009). Além de pesquisadores das universidades que compõem a rede desde o início, hoje integram também o grupo pesquisadores da Universidade de Ciências da Saúde de Alagoas, do Hospital de Reabilitação de Anomalias Craniofaciais da USP, da UNESP - Campi de Bauru e de Marília, e da Universidade Federal de Minas Gerais (UFMG). O tema "equivalência de estímulos e sua relação com comportamento simbólico" despertou o interesse também de pesquisadores da UFSC e da UEL, entre outras.

Três grupos de pesquisadores de diferentes universidades brasileiras têm se organizado e formalizado suas interações na Associação Brasileira de Pesquisa e Pós-Graduação (ANPEPP) por meio dos seguintes grupos de trabalho (GTs): Investigações conceituais e aplicadas em análise do comportamento, sob a coordenação de Maria Amália Andery; Análise comportamental de processos simbólicos, coordenado por Deisy das Graças de Souza; e Comportamento verbal e produção de tecnologias de intervenção e de prevenção em psicologia e educação, criado recentemente e coordenado por Verônica Bender Haydu.

Pode-se dizer que o maior número de contribuições de pesquisadores brasileiros em nível internacional até o momento são nos temas "escolha", "controle aversivo" e "equivalência de estímulos", com estudos publicados no JEAB (e.g., Todorov, 1971, 1973; Todorov, Castro Neto, Hanna, Sá \& Barreto, 1983; Todorov, Hanna \& Sá, 1984) e no Journal of Applied Behavior Analysis (JABA) (e.g., de Rose, de Souza \& Hanna, 1996; Melchiori, de Souza \& de Rose, 2000), os quais já foram citados diversas vezes. No entanto, temas mais recentemente estudados por pesquisadores com interação com universidades estrangeiras tem também merecido o reconhecimento nacional e internacional. Por exemplo, uma nova rede multi-institucional se formou nos últimos anos com o objetivo de desenvolver conceitualmente, descrever e estudar empiricamente práticas culturais, tendo como base os conceitos de metacontingências e macrocomportamentos. O grupo é composto por pesquisadores da PUC-SP, UFPA, UnB e University of North Texas (USA). Apesar de ser uma iniciativa recente, já existem artigos publicados em periódicos nacionais e internacionais (e.g., Andery \& Serio, 2003; Naves \& Vasconcelos, 2008; Todorov, 2005).

Nos anos 70 até meados dos anos 90 da década passada, era comum a realização de doutorado no exterior, quando os analistas do comportamento brasileiros tinham contato com pesquisadores de renome e experiência em outros temas, passavam a dominar novas metodologias e, no retorno, davam continuidade aos programas de pesquisa iniciados no exterior. Essa foi uma estratégia importante para ampliar as temáticas de pesquisa e a diversidade de conhecimento especializado no país, bem como aumentar a divulgação da produção nacional no exterior. São exemplos do resultado bem sucedido desse investimento a produção sobre: comportamento adjuntivo (e.g., Gimenes, Brandão \& Benvenuti, 2005); variabilidade comportamental (e.g., Abreu-Rodrigues, Lattal, Santos \& Matos, 2005), comportamento precorrente (e.g., Oliveira-Castro, Faria, Dias \& Coelho, 2002), modelo experimental de autocontrole (e.g., Hanna \& Todorov, 2002), comportamento de observação (e.g., Tomanari, Balsamo, Fowler, Farren \& Dube, 2007), para citar alguns. É nacionalmente reconhecida e aqui enfatizada também a qualidade da pesquisa desenvolvida pelo Grupo de Estudo e Pesquisa sobre Comportamento do Consumidor (e.g.,OliveiraCastro, Foxall \& Schrezenmaier, 2006) e dos trabalhos teóricoconceituais e empíricos desenvolvidos na PUC-SP (e.g., Andery, Micheletto \& Serio, 2005) e na UFPA (e.g., Tourinho, 2006).

A produção sobre análise comportamental aplicada com impacto internacional ainda é bastante escassa no Brasil. A maioria dos trabalhos aplicados tem sido desenvolvida em institutos privados de formação ${ }^{5}$, cujo número tem também crescido no país, mas sem o compromisso com a divulgação/ publicação. Essa é uma lacuna que uma comunidade tão grande deveria investir esforços para reduzir, especialmente devido à qualidade das muitas intervenções realizadas. Para melhorar o cenário da contribuição de impacto internacional, os institutos necessitam divulgar suas atividades e os resultados obtidos e/ou as universidades precisam assumir a tarefa de criar institutos voltados para a aplicação.

Os analistas do comportamento publicam ainda a maior parte de sua produção em periódicos nacionais. Pesquisas básicas e aplicadas e trabalhos de intervenção têm sido divulgados nas diversas revistas gerais de psicologia, especialmente Psicologia; Teoria e Pesquisa, Psicologia: Reflexão e Crítica, Temas em Psicologia e Psicologia (USP) e em revistas especializadas em análise do comportamento. $\mathrm{O}$ número de periódicos que publicam especificamente artigos teóricos e empíricos de análise do comportamento em língua portuguesa cresceu na última década. A primeira a ser criada foi a Revista Brasileira de Terapia Comportamental e Cognitiva, publicada pela ABPMC, criada por Rachel Kerbauy em 1999. A Revista Brasileira de Análise do Comportamento publica também artigos na língua inglesa e foi criada por João Claudio Todorov em 2005. Recentemente, o Núcleo Paradigma iniciou sua publicação intitulada Perspectiva em Análise do Comportamento. A Acta Comportamentalia é um periódico que publica artigos em línguas latinas da Universidad de Guadalajara que publica artigos em línguas latinas, do qual Maria Amélia Matos foi a primeira editora de

5 Diversos institutos têm oferecido cursos de formação de qualidade, suprindo lacunas dos cursos de psicologia em termos da aplicação da análise do comportamento. Na região sudeste, o pioneiro é o Instituto de Terapia por Contingências de Reforçamento, situado em Campinas e dirigido por Hélio Guilhardi. O Instituto Paradigma, em São Paulo, e o Instituto Brasiliense de Análise do Comportamento (IBAC), em Brasília, têm também se destacado nessa missão. 
língua portuguesa. A série Sobre Comportamento e Cognição, inicialmente organizada por Roberto Banaco, já se tornou um periódico e está em seu $24^{\circ}$ volume.

\section{Considerações Finais}

A introdução da análise do comportamento no Brasil impactou a psicologia em diversos aspectos. A psicologia foi reconhecida como profissão, laboratórios de pesquisa foram fundados e a formação do psicólogo, com a militância de nossos pioneiros, passou de puramente profissional para científica. Conselhos profissionais e sociedades científicas foram criados com a participação importante de analistas do comportamento. A organização de pesquisadores em redes multi-institucionais de psicologia tem reconhecimento no cenário nacional. Este texto retrata uma visão da história desse pioneirismo que, de certa forma, é consequência do contato com uma maneira de pensar o mundo e o comportamento, buscando entender e avaliar as interações humanas com ambientes complexos. Muitas pessoas e fatos importantes devem ter sido omitidos nessa versão da história. E uma versão que deve ser vista como uma complementação de vários outros relatos já publicados. Grande parte desses relatos são homenagens recebidas em vida (e.g., Feitosa, 2005; Guedes, 1998) e obituários sobre aqueles que nos deixaram precocemente (e.g., Batista, Ferrari, \& Laloni, 2005; Medeiros, 1999; Stamirowski, 1998; Tomanari, 2005). Esses relatos mostram que parte da vida e contribuições de grandes nomes de analistas do comportamento é a história do reconhecimento e estabelecimento da psicologia brasileira como ciência. Queremos destacar como forma de homenagear os(as) brasileiros(as) pioneiros(as) responsáveis pela psicologia e pela análise do comportamento de hoje ${ }^{6}$ : Carolina M. Bori†, Dora S. Fix Ventura, Isaias Pessotti, Margarida Windholz, Maria Amélia Matos†', Maria do Carmo Guedes, Maria Inês Rocha e Silva, Maria Lúcia D. Ferrara †, Maria Tereza Araújo, Rachel R. Kerbauy, Ricardo Gorayeb, Rodolpho Azzi e Sergio V. de Luna.

\section{Referências}

Abreu-Rodrigues, J., Lattal, K. A., Santos, C. V., \& Matos, R. A. (2005). Variation, repetition, and choice. Journal of the Experimental Analysis of Behavior, 83, 147-168.

6 Contribuíram também para a divulgação, criação de novos centros de pesquisa e formação e/ou para o desenvolvimento de conhecimento: Adélia M. S. Teixeira, Angela Duarte, Antonio Bento A. de Moraes, Antonio de Freitas Ribeiro, A. Celso N. Goyos, Caio Miguel, Carolina Lampréia, Célia M. L. C. Zannon, Deisy das Graças de Souza, Elenice Ferrari, Emmanuel Z. Tourinho, Hélio Guilhardi, Jorge M. OliveiraCastro, José Carlos Simões Fontes, José Lino O. Bueno, Josele Abreu Rodrigues, Julio C. de Rose, Kester Carrara, Laércia A. Vasconcelos, Ligia Marcondes Machado†t, Lincoln S. Gimenes, Lorismário E. Simonassi, Lúcia C. A. Williams, Luiz Marcelino de Oliveira†̣, Luiz Otávio de Seixas Queiroz†, Maly Delitti, Maria Amália Andery, Maria Helena Hunziker, Maria Martha Hübner, Maria Stella A. Gil, Nilza Micheletto, Olavo F. Galvão, Roberto Banaco, Silvio Botomé, Sonia B. Meyer, Tereza Maria A. Pires Serio†, Thereza P. L. Mettel. Pedimos desculpas por possíveis esquecimentos.
Andery, M. A., \& Sério, T. M. (2003). Metacontingencias y dialéctica: son incompatibles? Revista Lationamericana de Psicologia, 35, 273-280.

Andery, M. A. P. A., Micheletto, N., \& Sério, T. M. A. P. (2005). Meaning and verbal behavior in Skinner's work from 1934 to 1957. The Analysis of Verbal Behavior, 21, 163-174.

Ayllon, T., \& Michael, J. (1959). The psychiatric nurse as a behavioral engineer. Journal of the Experimental Analysis of Behavior, 2, 323-334.

Azzi, R., Fix, D. S. R., Keller, F. S., \& Rocha e Silva, M. I. (1964). Exteroceptive control of response under delayed reinforcement. Journal of the Experimental Analysis of Behavior, 7, 159-162.

Azzi, R., Rocha e Silva, M. I., Bori, C. M., Fix, D. S. R., \& Keller, F. 5. (1963). Suggested Portuguese translations of expressions in operant conditioning. Journal of the Experimental Analysis of Behavior, 6, 91-94.

Bachrach, A J. (1962). Experimental foundations of clinical psychology. New York: Basic Books.

Barros, R. S., \& Galvão, O. F. (2002). Generalized identity matching-to-sample in Cebus apella. The Psychological Record, 52, 441-460.

Batista, C. G., Ferrari, E. A. M., \& Laloni, D. T. (2005). Luiz Otávio de Seixas Queiroz: um pioneiro da análise do comportamento no Brasil. Revista Brasileira de Análise do Comportamento. 1, 269-273.

Baum, W. M. (1999). Understanding behaviorism: Science, behavior, and culture (M. T. A. Silva \& M. A. Matos Trads). Porto Alegre: Artmed. (Trabalho original publicado em 1994).

Biglan, A. (1995). Changing cultural practices: A contextualist framework for intervention research. Reno: Context Press.

Bori, C. M. (1996). Chapters in the life of Fred S. Keller. Psicologia: Teoria e Pesquisa, 12, 189-190.

Catania, A. C. (1999). Aprendizagem: comportamento, linguagem e cognição (D. G. Souza, Coord. Trad.). Porto Alegre: Artmed. (Trabalho original publicado em 1998)

de Rose, J. C., de Souza, D. G., \& Hanna, E. S. (1996). Teaching reading and spelling: Stimulus equivalence and exclusion. Journal of Applied Behavior Analysis, 29, 451-469.

de Souza, D. G., Moraes, A. B. A., \& Todorov, J. C. (1984). Shock intensity and signalled avoidance responding. Journal of the Experimental Analysis of Behavior, 42, 67-74.

Feitosa, M. A. G. (2005). Carolina Bori recebe o título de doutora honoris causa pela Universidade de Brasília. Revista Brasileira de Análise do Comportamento, 1, 263-267.

Ferrari, E. A. M., Todorov, J. C., \& de Souza, D. G. (1974). Key pecking as a function of response-shock and shock-shock intervals in unsignalled avoidance. Journal of the Experimental Analysis of Behavior, 22, 215-218.

Ferrari, E. A. M., Todorov, J. C., \& Graeff, F. G. (1973). Nondiscriminated avoidance of shocks by pigeons pecking a key. Journal of the Experimental Analysis of Behavior, 19, 211-218.

Gimenes, L. S., Brandão, A. M., \& Benvenuti, M. F. (2005). Comportamento adjuntivo: da pesquisa à aplicação. Em J. AbreuRodrigues \& M. R. Ribeiro (Orgs.), Análise do comportamento: pesquisa, teoria e aplicação (pp. 100-113). Porto Alegre: Artmed.

Glenn, S. S. (2003). Operant contingencies and the origin of cultures. Em K. A. Lattal \& P. N. Chase (Orgs.), Behavior theory and philosophy (pp. 223-242). New York: Kluver Academic / Plenum. 
Gorayeb, R. (1996). Introdução ao texto de Fred Keller "Imagens da vida de um professor". Psicologia: Teoria e Pesquisa, 12, 3-4.

Gorayeb, R., \& Todorov, J. C. (1977). Key pecking maintained by negative reinforcement: Multiple schedules. Revista Mexicana de Análisis de La Conducta, 3, 161-168.

Greenspoon, J. (1955). The reinforcing effect of two spoken sounds on the frequency of members of two verbal response classes. American Journal of Psychology, 68, 409-416.

Guedes, M. C. (1998). Carolina Bori: Retratos. Psicologia USP, 9, 225-236.

Guerin, B. (1994). Analyzing social behavior: Behavior analysis and the social sciences. Reno: Context Press.

Guilhardi, H., \& Madi, M. B. B. P. (1996). Professor Keller disse sim... Psicologia: Teoria e Pesquisa, 12, 113-114.

Hanna, E. S., \& Todorov, J. C. (2002). Modelos de autocontrole na análise experimental do comportamento: utilidade e crítica. Psicologia: Teoria e Pesquisa, 18, 337-343.

Harzem, P., \& Miles, T. R. (1978). Conceptual issues in operant psychology. Chichester: Wiley.

Herrnstein, J. R. (1970). On the law of effect. Journal of the Experimental Analysis of Behavior, 13, 243-266. (Tradução no prelo, Revista Brasileira de Análise do Comportamento, 4)

Honig, W.K. (1966). Introductory remaks. In W. K. Honing (Org.) Operante behavior: areas of research and application (pp. 1-11). Englewood Cliffs: Prentice-Hall.

Honig, W. K., \& Staddon, J. E. R. (1977). Handbook of operant behavior. Englewood Cliffs: Prentice-Hall.

Keller, F. S. (1968). Goodbye, teacher... Journal of Applied Behavior Analysis, 1, 78-89.

Keller, F. S. (1971, fevereiro). An international venture in behavior modification. II Symposium on Behavior Modification, Cidade do México, México.

Keller, F. S. (1981). Mulheres analistas do comportamento. Psicologia: Teoria e Pesquisa, 4, 43-46.

Keller, F. S. (1982). Pedagogue's progress. Lawrence: TRI.

Keller, F. S. (1987). O nascer de um departamento. Psicologia: Teoria e Pesquisa, 3, 198-205.

Keller, F. S., \& Schoenfeld, W. N. (1968). Principios de psicologia (C. M. Bori \& R. Azzi, Trads.). São Paulo: Herder. (Trabalho original publicado em 1950)

Kerbauy, R. R. (1983). Keller: o cientista ensina. Em R. R. Kerbauy (Org.) \& F. Fernandes (Coord.), Keller - Coleção Grandes Cientistas Sociais, Vol. 41 (pp. 7-42). São Paulo: Ática.

Lamal. P. (1997). Cultural contingencies: Behavior analytic perspectives on cultural practices. Westport, CT: Praeger.

Lattal, K. A., \& Chase, P. N. (2003). Behavior theory and phylosophy. New York: Kluwer Academic/Plenum.

Mace, D. A. (1948). Some implications of analytical behaviorism. Aristotelian Society, XLIX, 1-16.

Matos, M.A. (1996). Contingências para a análise comportamental no Brasil. Psicologia: Teoria e Pesquisa, 12, 107-111.

Matos, M. A. (1998). Carolina Bori: A psicologia brasileira como missão. Psicologia USP, 9, 67-70.

Medeiros, J. G. (1999). Em memória de Lígia Maria de Castro Marcondes Machado: homenagem póstuma à professora Lígia. Estudos de Psicologia (Campinas), 16, 65-67.

Melchiori, L. E., de Souza, D. G., \& de Rose, J. C. (2000). Reading, equivalence, and recombination of units: A replication with students with different learning histories. Journal of Applied Behavior Analysis, 33, 97-100.
Michael, J. (2004). Concepts and principles of behavior analysis. Kalamazoo: Association for Behavior Analysis International.

Millenson, J. R. (1967). Principles of behavioral analysis. New York: McMillan.

Moraes, A. B. A., Todorov, J. C. (1977). Signalled free-operant avoidance of shock by pigeons pecking a key. Journal of the Experimental Analysis of Behavior, 2, 27, 281-292.

Moreira, M. B., \& Medeiros, C. A. (2007). Princípios básicos de análise do comportamento. Porto Alegre: Artmed.

Naves, A. R. X. C., \& Vasconcelos, L. A. (2008). O estudo da família: contingências e metacontingências. Revista Brasileira de Análise do Comportamento, 4, 13-25.

Nazzaro, J. R., \& Todorov, J. C. (1966). Influence of luminance on a two choice decision task. Journal of Experimental Psychology: General. 71, 696-699.

Oliveira-Castro, J. M., Faria, J. B., Dias, M. B, \& Coelho, D. S. (2002). Effects of task complexity on learning to skip steps: An operant analysis. Behavioural Processes, 59, 101-120

Oliveira-Castro, J. M., Foxall, G. R., \& Schrezenmaier, T. C. (2006). Consumer brand choice: Individual and group analyses of demand elasticity. Journal of the Experimental Analysis of Behavior, 85, 147-166.

Orwell, G. (1949). 1984. New York: Harcourt, Brace.

Pessotti, I. (1996). Fred Keller, um mestre, meu mestre. Psicologia: Teoria e Pesquisa, 12, 1-2

Ribes-Iñesta, E. (2003). What is defined in operational definitions? The case of operant psychology. Behavior and Philosophy, 31, 111-126.

Robinson, G. (1977). Procedures for the acquisition of syntax. Em W. K. Honig \& J. E. R. Staddon (Orgs.), Handbook of operant behavior (pp. 619-627). Englewood Cliffs: Prentice-Hall,

Schick, K. (1971). Operants. Journal of the Experimental Analysis of Behavior, 16, 413-423.

Schwartz, B., \& Gamzu, E. (1977). Pavlovian control of operant behavior. Em W. K. Honing \& J. E. R. Staddon (Orgs.), Handbook of operant behavior (pp. 53-97). Englewood Cliffs: Prentice-Hall.

Segal, E. (1977). Toward a coherent psychology of language. Em W. K. Honing \& J. E. R. Staddon (Orgs.), Handbook of operant behavior (pp. 628-654). Englewood Cliffs: Prentice-Hall.

Sidman, M. (1998). Coercion and its fallout (M. A. Andery \& T. M. Sério, Trads.). Campinas: Editorial Psy. (Trabalho original publicado em 1989)

Sidman, M., \& Tailby, W. (1982). Conditional discrimination vs. matching to sample: An expansion of the testing paradigm. Journal of the Experimental Analysis of Behavior, 37, 5-22.

Skinner, B. F. (1938). The behavior of organisms. New York: Appleton-Century-Crofts.

Skinner, B. F. (1961). Cumulative record. New York: AppletonCentury-Crofts.

Skinner, B. F. (1967). Ciência e comportamento humano (J. C. Todorov \& R. Azzi, Trads.). Brasília: Universidade de Brasília. (Trabalho original publicado em 1953)

Skinner, B. F. (1974). About behaviorism. New York: Alfred A. Knopf.

Skinner, B. F. (1978). O comportamento verbal (M. P. Villalobos, Trad.). São Paulo: Cultrix. (Trabalho original publicado em 1957)

Skinner, B. F. (1980). Contingências do reforço: uma análise teórica - Coleção Os Pensadores (R. Moreno, Trad.). São Paulo: Abril Cultural. (Trabalho original publicado em 1969) 
Souza, C. B. A., Borges, R. P., Goulart, P. R. K., Barros, R. S., \& Galvão, O. F. (2009). Testes de identidade generalizada com objetos em macaco-prego (Cebus apella). Psicologia: Teoria e Pesquisa, 25, 169-177.

Staats, A. W., Staats, C. K., Schultz, R. E., \& Wolf M. (1962). The conditioning of textual responses using "extrinsic" reinforcers. Journal of the Experimental Analysis of Behavior, 5, 33-40.

Staddon, J.E.R. (1973). On the notion of cause with applications to behaviorism. Behaviorism, 1, 25-63.

Stamirowski, O. L. (1998). Prolegômeros ao conhecimento de uma Carolina Bori. Psicologia USP, 9, 251-260.

Todorov, J. C. (1971). Concurrrent performances: Effect of punishment contingent on the switching response. Journal of the Experimental Analysis of Behavior, 16, 51-62.

Todorov, J. C. (1973). Interaction of frequency and magnitude of reinforcement on concurrent performances. Journal of the Experimental Analysis of Behavior, 19, 451-458.

Todorov, J. C. (1977). Effects of punishment of main-key responding in concurrent schedules. Revista Mexicana de Análisis de La Conduta, 3, 17-28.

Todorov, J. C. (1979). Neglected operants in concurrent performances. Revista Mexicana de Análisis de La Conduta, 5, 21-26.

Todorov, J. C. (1981). Behavior analysis and experimental pharmacology. Neuroscience and Biobehavioral Reviews, 5, 307-314.

Todorov, J. C. (1982). Behaviorismo e análise experimental do comportamento. Cadernos de Análise do Comportamento, 3, 10-23.

Todorov, J. C. (1983). Estados de transição e estados transitórios. Anais da XIII Reunião Anual de Psicologia, 13, 327-342.

Todorov, J. C. (1987). A constituição como metacontingência. Psicologia Ciência e Profissão, 7, 9-13.

Todorov, J. C. (1989). A psicologia como estudo de interações. Psicologia: Teoria e Pesquisa, 5, 325-347. (Trabalho republicado em 2007, Psicologia: Teoria e Pesquisa, 23, 57-61)

Todorov, J. C. (1990). The K\&S in Brazil. Journal of the Experimental Analysis of Behavior, 54, 151-152.

Todorov, J. C. (1991). O conceito de contingência na psicologia experimental. Psicologia: Teoria e Pesquisa, 7, 59-70.

Todorov, J. C. (1996). Goodbye teacher, good old friend. Journal of the Experimental Analysis of Behavior, 66, 7-9.
Todorov, J. C. (2003). Science and Human Behavior translated into Portuguese: Ciência e Comportamento Humano. Journal of the Experimental Analysis of Behavior, 80, 341-343.

Todorov, J. C. (2005). Laws and the complex control of behavior. Behavior and Social Issues, 14, 86-91.

Todorov, J. C. (2006). Behavior analysis in Brasil. Avances en Psicologia Latinoameticana, 24, 29-36.

Todorov, J. C., Castro Neto, J. M. O., Hanna, E. S., Sá, M. C. N. B., \& Barreto, M. Q. (1983). Choice, experience and the generalized matching law. Journal of the Experimental Analysis of Behavior, 10, 99-111.

Todorov, J. C., Hanna, E. S., \& Sá, M. C. N. B. (1984). Frequency versus magnitude of reinforcement: New data with a different procedure. Journal of the Experimental Analysis of Behavior, 41, 157-167.

Todorov, J. C., \& Moreira, M. (2004). Análise experimental do comportamento e sociedade: um novo foco de estudo. Psicologia: Reflexão e Crítica, 17, 25-29.

Todorov, M. S. R. (1995). UnB: evolução da estrutura acadêmica. Brasília: Universidade de Brasília.

Todorov, M. S. R. (1997). A psicologia na Universidade de Brasília. Brasília: Universidade de Brasília.

Tomanari, G. Y. (2005). Notícia: Maria Amelia Matos (19392005): generosidade, competência, liderança. Psicologia: Teoria e Pesquisa, 21, 255-256.

Tomanari, G. Y., Balsamo, L. M., Fowler, T. R., Farren, K. M., \& Dube, W. V. (2007). Manual and ocular observing behavior in human subjects. European Journal of Behavior Analysis, 8, 29-40.

Tourinho, E. Z. (2006). Private stimuli, covert responses and private events: Conceptual remarks. The Analysis of Verbal Behavior, 29, 13-31.

Weingarten, K., \& Mechner, F. (1966). The contingency as an independent variable of social interaction. Em T. Verhave (Org.), Readings in the experimental analysis of behavior (pp. 447-459). New York: Appleton-Century-Crofts.

Zannon, C. M. L. C. (1996). Editorial: um tributo a Fred Keller. Psicologia: Teoria e Pesquisa, 12, 3-4.

Zannon, C. M. L. C., \& Bori (1996). SBPC 1972 - Relato do Plano Brasília por Fred S. Keller. Psicologia: Teoria e Pesquisa, 12, 191-192. 